NTUA-98-2000

hep-th/0010014

\title{
Cosmological Evolution of a Brane Universe in a Type 0 String Background.
}

\author{
E. Papantonopoulos ${ }^{a}$ and I. Pappa ${ }^{b}$ \\ National Technical University of Athens, Physics Department, Zografou Campus, \\ GR 157 80, Athens, Greece.
}

\begin{abstract}
We study the cosmological evolution of a D3-brane Universe in a type 0 string background. We follow the brane-universe along the radial coordinate of the background and we calculate the energy density which is induced on the brane because of its motion in the bulk. We find that for some typical values of the parameters and for a particular range of values of the scale factor of the brane-universe, the effective energy density is dominated by a term proportional to $\frac{1}{(\log a)^{4}}$ indicating a slow varying inflationary phase. For larger values of the scale factor the effective energy density takes a constant value and the brane-universe enters its usual inflationary period.
\end{abstract}

${ }^{a}$ e-mail address:lpapa@central.ntua.gr

${ }^{b}$ e-mail address:gpappa@central.ntua.gr 


\section{Introduction}

There has been much recent interest in the idea that our universe may be a brane embedded in some higher dimensional space [1]. It has been shown that the hierarchy problem can be solved if the higher dimensional Planck scale is low and the extra dimensions large [2]. Randall and Sundrum [3] proposed a solution of the hierarchy problem without the need for large extra dimensions but instead through curved five-dimensional spacetime $A d S_{5}$ that generates an exponential suppression of scales.

This idea of a brane-universe can naturally be applied to string theory. In this context, the Standard Model gauge bosons as well as charged matter arise as fluctuations of the Dbranes. The universe is living on a collection of coincident branes, while gravity and other universal interactions is living in the bulk space [⿴囗十). For example, the strongly coupled $E_{8} \times E_{8}$ heterotic string theory is believed to be an eleven-dimensional theory, the field theory limit of which was described by Horava and Witten [5]. The spacetime manifold includes a compact dimension with an orbifold structure. Matter is confined on the two ten-dimensional hypersurfaces (nine-branes) which can be seen as forming the boundaries of this spacetime.

This new concept of brane-universe naturally leads to a new approach to cosmology. Any cosmological evolution like inflation has to take place on the brane while gravity acts globally on the whole space. In the literature there are a lot of cosmological models which study the cosmological evolution of our universe. In most of these models the spacetime is five-dimensional, where the fifth dimension is the radial dimension of an $A d S_{5}$ space. The effective Einstein equations on the brane are then solved taking under consideration the matter on the brane [6]- 9$]$.

Another approach to cosmological evolution of our brane-universe is to consider the

motion of the brane in higher dimensional spacetimes. In [8] the motion of a domain wall (brane) in such a space was studied. The Israel matching conditions were used to relate 
the bulk to the domain wall (brane) metric, and some interesting cosmological solutions were found. In [9] a universe three-brane is considered in motion in ten-dimensional space in the presence of a gravitational field of other branes. It was shown that this motion in ambient space induces cosmological expansion (or contraction) on our universe, simulating various kinds of matter.

In this direction we have studied [10], the motion of a three-brane in the background of type 0 string theory . It was shown that the motion of the brane on this specific background, with constant values of dilaton and tachyon fields, induces a cosmological evolution which for some range of the parameters has an inflationary phase. In [11], using similar technics the cosmological evolution of the three-brane in the background of type IIB string theory was considered.

Type 0 string theories [13] are interesting because of their connection [15] to fourdimensional $S U(N)$ gauge theory. The type 0 string does not have spacetime supersymmetry and because of that contains in its spectrum a non-vanishing tachyon field. In [13] it was argued that one could construct the dual of an $\mathrm{SU}(\mathrm{N})$ gauge theory with 6 real adjoint scalars by stacking $\mathrm{N}$ electric three-dimensional branes of the type 0 model on top of each other. The tachyon field couples to the five form field strength, which drives the tachyon to a nonzero expectation value.

Asymptotic solutions of the dual gravity background were constructed in [13, 20]. At large radial coordinate the tachyon is constant and one finds a metric of the form $A d S_{5} \times S^{5}$ with vanishing coupling which was interpreted as a UV fixed point. The solution exhibits a logarithmic running in qualitative agreement with the asymptotic freedom property of the field theory. At small radial coordinate the tachyon vanishes and one finds again a solution of the form $A d S_{5} \times S^{5}$ with infinite coupling, which was interpreted as a strong coupling IR fixed point. A gravity solution which describes the flow from the UV fixed point to the IR fixed point is given in 12 . 
From the holographic principle and the AdS/CFT correspondence, this renormalization group flow of type 0 string theory, can be understood to correspond to moving the brane a finite distance in the bulk. Motivated by this behaviour of type 0 theory, we will study the cosmological evolution of the brane-universe as the brane moves from the UV to the IR fixed point.

We calculate the effective energy density which is induced on the brane because of its motion in the particular background of a type 0 string. Using the approximate solutions of 13 , 20, 12], we find that for large values of the radial coordinate $r$, in the UV region, the effective energy density takes a constant value, which means that the universe has an inflationary period. For smaller values of $r$, or of the scale factor $\alpha$, the energy density is dominated by a term proportional to $\frac{1}{(\log a)^{4}}$, where $\alpha$ is the scale factor of the brane-universe. This value of the energy density indicates that the universe is in a slow inflationary phase, in a "logarithmic inflationary" phase as we can call it, in contrast to "constant inflationary" phase which characterizes the usual exponential behaviour. For even smaller values of $r$, the approximation breaks down and we cannot trust anymore the solutions. If we go to the IR region the energy density is dominated by the term $\frac{1}{\alpha^{4}}$ and again we find the "logarithmic inflation" for larger values of $r$. The approximation breaks down again for some larger values of $r$. It is well known that it is very difficult to connect the IR to the UV solutions. Therefore our failure to present a full cosmological evolution, relies exactly on this fact [14].

We note here that what we find is somewhat peculiar, in the sense that one does not expect the effective energy density to be dominated, for a range of values of the scale factor, by terms proportional to $\frac{1}{(\log a)^{4}}$. We understand this behaviour, as due entirely to mirage matter which is induced on the brane, from this particular background.

Our work is organized as follows. In section two, we develop the formalism for a brane moving in a string background with a dilaton and a RR field. In section three, we discuss type 0 string and except the exact solution in an $A d S_{5} \times S^{5}$ background we discuss the 
asymptotic UV and IR solutions of type 0 strings. In section four, we discuss the cosmological evolution of a brane in the background of type 0 string. Finally in the last section we discuss our results.

\section{Brane moving in ten-dimensional background}

We will consider a probe brane moving in a generic static, spherically symmetric background [9]. The brane will move in a geodesic. We assume the brane to be light compared to the background so that we will neglect the back-reaction. As the brane moves the induced world-volume metric becomes a function of time, so there is a cosmological evolution from the brane point of view. The metric of a D3-brane is parametrized as

$$
d s_{10}^{2}=g_{00}(r) d t^{2}+g(r)(d \vec{x})^{2}+g_{r r}(r) d r^{2}+g_{S}(r) d \Omega_{5}
$$

and there is also a dilaton field $\Phi$ as well as a $R R$ background $C(r)=C_{0 \ldots 3}(r)$ with a selfdual field strength. The dynamics on the brane will be governed by the Dirac-Born-Infeld action given by

$$
\begin{aligned}
S= & T_{3} \int d^{4} \xi e^{-\Phi} \sqrt{-\operatorname{det}\left(\hat{G}_{\alpha \beta}+\left(2 \pi \alpha^{\prime}\right) F_{\alpha \beta}-B_{\alpha \beta}\right)} \\
& +T_{3} \int d^{4} \xi \hat{C}_{4}+\text { anomaly terms }
\end{aligned}
$$

The induced metric on the brane is

$$
\hat{G}_{\alpha \beta}=G_{\mu \nu} \frac{\partial x^{\mu} \partial x^{\nu}}{\partial \xi^{\alpha} \partial \xi^{\beta}}
$$

with similar expressions for $F_{\alpha \beta}$ and $B_{\alpha \beta}$. In the static gauge, $x^{\alpha}=\xi^{\alpha}, \alpha=0,1,2,3$ using (2.3) we can calculate the bosonic part of the brane Lagrangian which reads

$$
L=\sqrt{A(r)-B(r) \dot{r}^{2}-D(r) h_{i j} \dot{\varphi}^{i} \dot{\varphi}^{j}}-C(r)
$$


where $h_{i j} d \varphi^{i} d \varphi^{j}$ is the line element of the unit five-sphere, and

$$
A(r)=g^{3}(r)\left|g_{00}(r)\right| e^{-2 \Phi}, B(r)=g^{3}(r) g_{r r}(r) e^{-2 \Phi}, D(r)=g^{3}(r) g_{S}(r) e^{-2 \Phi}
$$

and $C(r)$ is the $R R$ background. Demanding conservation of energy $E$ and of total angular momentum $\ell^{2}$ on the brane, we find

$$
\dot{r}^{2}=\frac{A}{B}\left(1-\frac{A}{(C+E)^{2}} \frac{D+\ell^{2}}{D}\right), h_{i j} \dot{\varphi}^{i} \dot{\varphi}^{j}=\frac{A^{2} \ell^{2}}{D^{2}(C+E)^{2}}
$$

We can see that the above relation gives the following constraint

$$
\left(1-\frac{A}{(C+E)^{2}} \frac{D+\ell^{2}}{D}\right) \geq 0
$$

The induced four-dimensional metric on the brane is

$$
d \hat{s}^{2}=\left(g_{00}+g_{r r} \dot{r}^{2}+g_{S} h_{i j} \dot{\varphi}^{i} \dot{\varphi}^{j}\right) d t^{2}+g(d \vec{x})^{2}
$$

In the above relation we substitute $\dot{r}^{2}$ and $h_{i j} \dot{\varphi}^{i} \dot{\varphi}^{j}$ from (2.6), and we get

$$
d \hat{s}^{2}=-d \eta^{2}+g(r(\eta))(d \vec{x})^{2}
$$

with $\eta$ the cosmic time which is defined by

$$
d \eta=\frac{\left|g_{00}\right| g^{\frac{3}{2}} e^{-\Phi}}{|C+E|} d t
$$

Having the induced metric (2.9) on the brane, which is the standard form of a flat expanding universe, we can write the Einstein equations on the brane,

$$
R_{\mu \nu}-\frac{1}{2} g_{\mu \nu} R=8 \pi G T_{\mu \nu}-\Lambda g_{\mu \nu}
$$

where $T_{\mu \nu}$ is the energy momentum tensor and $\Lambda$ the cosmological constant, is a function of cosmic time $\eta$. Taking for the energy momentum tensor the usual form of a perfect fluid, the Einstein equations become,

$$
8 \pi G \rho+\Lambda=\frac{3}{4} g^{-2} \dot{g}^{2}
$$




$$
\begin{gathered}
8 \pi G p+\Lambda=g^{-1} \ddot{g}-\frac{1}{4} g^{-2} \dot{g}^{2} \\
8 \pi G \dot{\rho}+\frac{3}{2} 8 \pi G g^{-1} \dot{g}(p+\rho)+\dot{\Lambda}=0
\end{gathered}
$$

Equation (2.14) is coming from the conservation of energy momentum tensor $T^{\mu \nu}{ }_{\nu}$. If we supplement the above equations with an equation of state

$$
p=(\gamma-1) \rho
$$

we have the set of equations which give the cosmological evolution of the brane-universe. All the quantities in these equations are functions of cosmic time as this time is measured by an observer on the brane. In return because of (2.10), and for the consistency of our scheme, $p, \rho$, and $\Lambda$ become functions of the radial coordinate $\mathrm{r}$. Therefore equations (2.12) - 2.15) act as effective cosmological equations induced by the bulk background fields. We can then identify the left hand side of (2.12) with $\rho_{\text {eff }}$

$$
8 \pi G \rho+\Lambda=8 \pi G \rho_{e f f}
$$

in the sense that the cosmological evolution described by this equation is driven by bulk background fields through a "mirage" matter $\rho_{\text {eff }}$ which is induced on the brane. Using equation (2.10) we get

$$
\dot{g}=g^{\prime}\left[\frac{\left|g_{00}\right|}{g_{r r}}-\frac{g_{00}^{2}}{g_{s} g_{r r}}\left(\frac{g^{3} g_{s} e^{-2 \Phi}+\ell^{2}}{(C+E)^{2}}\right)\right]^{\frac{1}{2}} \frac{|C+E|}{\left|g_{00}\right| g^{\frac{3}{2}} e^{-\Phi}}
$$

where prime denotes differentiation with respect to $r$. If we define the scale factor as $g=\alpha^{2}$ then equation (2.16) with the use of (2.12) and (2.17) becomes

$$
\frac{8 \pi}{3} \rho_{e f f}=\left(\frac{\dot{\alpha}}{\alpha}\right)^{2}=\frac{(C+E)^{2} g_{S} e^{2 \Phi}-\left|g_{00}\right|\left(g_{S} g^{3}+\ell^{2} e^{2 \Phi}\right)}{4\left|g_{00}\right| g_{r r} g_{S} g^{3}}\left(\frac{g^{\prime}}{g}\right)^{2}
$$

Therefore the motion of a D3-brane on a general spherically symmetric background had induced on the brane a matter density. As it is obvious from the above relation, the specific form of the background will determine the cosmological evolution on the brane. 


\section{Type 0 string background}

Type 0 string theory is interesting because of its connection to gauge theories. This enables us to study $\mathrm{SU}(\mathrm{N})$ gauge theory by merely gravitational quantities. Another advantage of this theory is the presence of a tachyon field. Tachyonic fields in ordinary field theory create instabilities. In cosmology on the contrary, the time evolution of a tachyon field plays an important rôle. In two dimensions because the tachyon field is a matter field has important consequences in cosmology [17, and it can give a solution to the "gracefull exit" problem [18]. In four dimensions its effect to cosmology has been examined by various authors [19].

As we have shown in [10] in type 0 string the tachyon field can induce inflation on the brane. We had used an exact solution of type 0 string with constant tachyon and dilaton fields. If these fields are coordinate dependent then, there is not an exact solution of the theory, but there are approximate solutions which we will discuss in the following. The action of the type 0 string is given by 13

$$
\begin{aligned}
S_{10}= & \int d^{10} x \sqrt{-g}\left[e^{-2 \Phi}\left(R+4\left(\partial_{\mu} \Phi\right)^{2}-\frac{1}{4}\left(\partial_{\mu} T\right)^{2}-\frac{1}{4} m^{2} T^{2}-\frac{1}{12} H_{\mu \nu \rho} H^{\mu \nu \rho}\right)\right. \\
& \left.-\frac{1}{4}\left(1+T+\frac{T^{2}}{2}\right)\left|F_{5}\right|^{2}\right]
\end{aligned}
$$

The equations of motion which result from this action, with the antisymmetric field put to zero, are

$$
\begin{gathered}
2 \nabla^{2} \Phi-4\left(\nabla_{n} \Phi\right)^{2}-\frac{1}{4} m^{2} T^{2}=0 \\
R_{m n}+2 \nabla_{m} \nabla_{n} \Phi-\frac{1}{4} \nabla_{m} T \nabla_{n} T-\frac{1}{4 \cdot 4 !} e^{2 \Phi} f(T)\left(F_{m k l p q} F_{n}{ }^{k l p q}\right. \\
\left.-\frac{1}{10} G_{m n} F_{s k l p q} F^{s k l p q}\right)=0
\end{gathered}
$$




$$
\begin{gathered}
\left(-\nabla^{2}+2 \nabla^{n} \Phi \nabla_{n}+m^{2}\right) T+\frac{1}{2 \cdot 5 !} e^{2 \Phi} f^{\prime}(T) F_{s k l p q} F^{s k l p q}=0 \\
\nabla_{m}\left(f(T) F^{\text {mnkpq }}\right)=0
\end{gathered}
$$

The tachyon is coupled to the $R R$ field through the function

$$
f(T)=1+T+\frac{1}{2} T^{2}
$$

In the background where the tachyon field acquires vacuum expectation value $T_{\text {vac }}=T_{0}=$ -1 , the tachyon function (3.6) takes the value $f\left(T_{0}\right)=\frac{1}{2}$ which guarantee the stability of the theory 13 .

The equations (3.2)-(3.5) can be solved using the following ansatz for the metric

$$
d s_{10}^{2}=g_{00}(r) d t^{2}+g(r)(d \vec{x})^{2}+g_{r r}(r) d r^{2}+g_{S}(r) d \Omega_{5}
$$

Technically it is easier to solve the above equations if we go to new variables. One can then introduce new parameter $\rho$ through the relation

$$
\rho=\frac{e^{2 \Phi_{0}}}{4 r^{4}}
$$

and the fields $\xi$ and $\eta$ from the relations

$$
\begin{gathered}
g=e^{\frac{\Phi-\xi}{2}} \\
g_{s}=e^{\frac{\Phi+\xi}{2}-\eta}
\end{gathered}
$$

Then (3.7) takes the form, 


$$
d s^{2}=-e^{\frac{\Phi-\xi}{2}} d t^{2}+e^{\frac{\Phi-\xi}{2}} d \vec{x}^{2}+e^{\frac{\Phi+\xi}{2}-5 \eta} d \rho^{2}+e^{\frac{\Phi+\xi}{2}-\eta} d \Omega_{5}^{2}
$$

With this form of the metric the action (3.1) can be described by the following Toda-like mechanical system (dot denotes $\rho$-derivative)

$$
S=\int d \rho\left[\frac{1}{2} \dot{\Phi}^{2}+\frac{1}{2} \dot{\xi}^{2}+\frac{\dot{T}^{2}}{4}-5 \dot{\eta}^{2}-V(\Phi, \xi, T, \eta)\right]
$$

with the potential $V(\Phi, \xi, T, \eta)$ given by

$$
V(\Phi, \xi, T, \eta)=g(T) e^{\frac{1}{2} \Phi+\frac{1}{2} \xi-5 \eta}+20 e^{-4 \eta}-Q^{2} f^{-1}(T) e^{-2 \xi}
$$

If the tachyon field takes its vacuum value and the dilaton field a constant value $\Phi=\Phi_{0}$ one can find the electrically charged three-brane

$$
g_{00}=-H^{-\frac{1}{2}}, g(r)=H^{-\frac{1}{2}}, g_{S}(r)=H^{\frac{1}{2}} r^{2}, g_{r r}(r)=H^{\frac{1}{2}}, H=1+\frac{e^{\Phi_{0}} Q}{2 r^{4}}
$$

if the following ansatz for the $R R$ field

$$
C_{0123}=A(r), F_{0123 r}=A^{\prime}(r)
$$

is used.

If $T$ and $\Phi$ are functions of the coordinate $r$, then approximate solutions exist [13, 20]. These solutions are valid for large (UV) and small (IR) values of the radial coordinate. The approximations of [13, 20] agree in the UV region but in the IR the approximation of [13] leads to an IR fixed point, while the approximation of [20] to a confining point.

From the action (3.12) we can derive the following equations of motion [20]

$$
\ddot{\xi}+\frac{1}{2} g(T) e^{\frac{1}{2} \Phi+\frac{1}{2} \xi-5 \eta}+2 \frac{Q^{2}}{f(T)} e^{-2 \xi}=0
$$




$$
\begin{gathered}
\ddot{\eta}+\frac{1}{2} g(T) e^{\frac{1}{2} \Phi+\frac{1}{2} \xi-5 \eta}+8 e^{-4 \eta}=0 \\
\ddot{\Phi}+\frac{1}{2} g(T) e^{\frac{1}{2} \Phi+\frac{1}{2} \xi-5 \eta}=0 \\
\ddot{T}+2 g^{\prime}(T) e^{\frac{1}{2} \Phi+\frac{1}{2} \xi-5 \eta}+2 \frac{Q^{2} f^{\prime}(T)}{f^{2}(T)} e^{-2 \xi}=0
\end{gathered}
$$

where $g(T)$ is the bare tachyon potential,

$$
g(T)=\frac{1}{2} T^{2}-\lambda T^{4}
$$

and $\lambda$ is a parameter. Defining a new variable $\rho=u^{-4}$, in the UV for $u \longrightarrow \infty$, or $\rho \longrightarrow 0$, we can solve the equations of motion (3.16)-(3.19) to the next to leading order and find 20, 13

$$
\begin{gathered}
T=T_{0}-4 \frac{g^{\prime}\left(T_{0}\right)}{g\left(T_{0}\right)} \frac{1}{\log \rho}+O\left(\frac{\log (-\log \rho)}{\log ^{2} \rho}\right) \\
\Phi=-2 \log \left(C_{0} \log \rho\right)-\left(7+8\left(\frac{g^{\prime}\left(T_{0}\right)}{g\left(T_{0}\right)}\right)^{2}\right)\left(\frac{\log (-\log \rho)}{\log \rho}\right)+\frac{B}{\log \rho}+O\left(\frac{\log ^{2}(-\log \rho)}{\log ^{2} \rho}\right) \\
\xi=\log \left(\sqrt{2 f^{-1}\left(T_{0}\right)} Q \rho\right)-\frac{1}{\log \rho}+O\left(\frac{\log (-\log \rho)}{\log \rho}\right) \\
\eta=\frac{1}{2} \log (4 \rho)-\frac{1}{\log \rho}+O\left(\frac{\log ^{2}\left(-\log ^{2} \rho\right)}{\log ^{2} \rho}\right)
\end{gathered}
$$

where $C_{0}=-\frac{4 C_{2}^{5}}{g\left(T_{0}\right) \sqrt{ } C_{1}}$ and

$$
C_{1}=\frac{2 Q}{\sqrt{2 f\left(T_{0}\right)}}\left(1+\frac{1}{4 \log \frac{u}{u_{0}}}\right), C_{2}=2\left(1+\frac{1}{4 \log \frac{u}{u_{0}}}\right)
$$


The above solutions show that at the UV point, the tachyon takes a constant value and if we calculate the next to leading order effective coupling $e^{\frac{1}{2} \Phi}$,

$$
e^{\frac{1}{2} \Phi} \sim \frac{1}{\log u-\left(\frac{7}{8}+\frac{g^{\prime}\left(T_{0}\right)^{2}}{g\left(T_{0}\right)^{2}}\right) \log \log u}
$$

we see that goes to zero for large $u$.

For $u \longrightarrow 0$ or for large $\rho$ there are two approximate solutions in the literature, leading to infrared fixed point [13] or to a confining fixed point [20, 12]. For the first approximation we have

$$
\begin{gathered}
T=-\frac{16}{\log \rho}-\frac{8}{\log ^{2} \rho}(9 \log \log \rho-3)+O\left(\frac{\log ^{2} \log \rho}{\log ^{3} \rho}\right) \\
\Phi=-\frac{1}{2} \log \left(2 Q^{2}\right)+2 \log \log \rho-\frac{1}{\log \rho} 9 \log \log \rho+O\left(\frac{\log \log \rho}{\log ^{2} \rho}\right) \\
\xi=\frac{1}{2} \log \left(2 Q^{2}\right)+\log \rho+\frac{9}{\log \rho}+\frac{9}{2 \log ^{2} \rho}\left(9 \log \log \rho-\frac{20}{9}\right)+O\left(\frac{\log ^{2} \log \rho}{\log ^{3} \rho}\right) \\
\eta=\log 2+\frac{1}{2} \log \rho+\frac{1}{\log \rho}+\frac{1}{2 \log ^{2} \rho}(9 \log \log \rho-2)+O\left(\frac{\log ^{2} \log \rho}{\log ^{3} \rho}\right)
\end{gathered}
$$

While for the second solution [20, 12] we have

$$
\begin{gathered}
\Phi=\Phi_{0}+\rho-\frac{1}{16(\sqrt{5}+3)^{2}} e^{\frac{\Phi_{0}}{2}} e^{-(\sqrt{5}+3) \rho} \\
\eta=\frac{1}{\sqrt{5}} \rho-\frac{5}{2} e^{-\frac{4}{\sqrt{5}} \rho} \\
\xi=\rho-\frac{1}{2} e^{-2 \rho}
\end{gathered}
$$




$$
T=-\frac{1}{2} e^{-2 \rho}
$$

In both approximations the tachyon field in the IR point goes to zero while the effective coupling gets infinite. It is important to observe that the approximate solutions in the UV (3.21)-(3.24) and in the IR (3.28)-(3.30) of 13 are related by $y \longrightarrow-y$, suggesting that they can be smoothly connected into a full interpolating solution. An attempt to connect these solutions was presented in [12]. As we mention above, the tachyon field starts at $\mathrm{T}=-1$ at $\rho=0$ in the UV, and grows according to (3.21), then enters an oscillating regime and finally relaxes to zero according to (3.27), when $\rho=\infty$ in IR. This guarantees that $T^{2} e^{\frac{1}{2} \Phi}$ becomes small which leads the metric in the $A d S_{5} \times S^{5}$ form.

There is a question if we can trust the asymptotic solutions in the infrared. The problem is that when the coupling becomes strong, string corrections become important. The situation is different in the UV where we can trust our solutions because the coupling is small. The role of the $\alpha^{\prime}$ corrections in the IR has been discussed in [20, 12]. It was claimed that the $\alpha^{\prime}$ corrections are small.

\section{Cosmological evolution of the Brane-Universe}

We consider a D3-brane moving along a geodesic in the background of a type 0 string. Having all the solutions in the ultra violet and the infrared, we can follow the cosmological evolution of our universe as it moves along the radial coordinate $r$. In the presence of a non trivial tachyon field the coupling $e^{-\Phi}$ which appears in the Dirac-Born-Infeld action in (2.2), is modified by a tachyonic function $\kappa(T)=1+\frac{1}{4} T+O\left(T^{2}\right)$. Then we can define an effective coupling [15]

$$
e_{e f f}^{-\Phi}=\kappa(T) e^{-\Phi}
$$

The bulk fields are also coordinate dependent and the induced metric on the brane 
will depend on a non trivial way on the dilaton field. Therefore the metric in the string frame will be connected to the metric in the Einstein frame through $g_{S t}=e_{e f f}^{\frac{\Phi}{2}} g_{E}$. All the quantities used so far were defined in the string frame. We will follow our cosmological evolution in the Einstein frame. Then the relation (2.18) becomes

$$
\frac{8 \pi}{3} \rho_{e f f}=\left(\frac{\dot{\alpha}}{\alpha}\right)^{2}=\frac{(C+E)^{2} g_{S}-\left|g_{00}\right|\left(g_{S} g^{3}+\ell^{2}\right)}{4\left|g_{00}\right| g_{r r} g_{S} g^{3}}\left(\frac{g^{\prime}}{g}\right)^{2}
$$

Having the approximate solution in the UV given by (3.21)-(3.24) we can calculate the metric components of the metric (3.7) and find

$$
\begin{gathered}
g_{y y}=\frac{1}{16} \sqrt{\frac{Q}{2}}\left(1-\frac{9}{2 y}\right) \\
g=\frac{1}{\sqrt{2 Q}} e^{\frac{y}{2}}\left(1-\frac{1}{2 y}\right) \\
g_{s}=\sqrt{\frac{Q}{2}}\left(1-\frac{1}{2 y}\right)
\end{gathered}
$$

The variable $\mathrm{y}$ is defined by

$$
\rho=e^{-y}
$$

Then we can identify $g$ of (4.4) with the scale factor $\alpha^{2}$ and solve for $y$. We get two solutions

$$
\begin{gathered}
y_{1}=-\frac{1}{4 \log \alpha+\log 2 Q} \\
y_{2}=\log 2 Q+4 \log \alpha+\frac{1}{\log 2 Q+4 \log \alpha}
\end{gathered}
$$


From the solution (4.8) which has the right behaviour for large $\alpha$, we keep the $\log 2 Q+$ $4 \log \alpha$ term. Then the $R R$ field $\mathrm{C}$ becomes

$$
C=\frac{e^{y}}{Q}-\frac{2}{Q} E i[y]
$$

Then, we can calculate the effective energy density from (4.2) setting $\ell^{2}=0$ and we get

$$
\begin{aligned}
\frac{8 \pi}{3} \rho_{\text {eff }}= & {\left[\left(1-\frac{1}{Q \alpha^{4}} \operatorname{Ei}[\log 2 Q+4 \log \alpha]+\frac{E}{2 \alpha^{4}}\right)^{2}\right.} \\
& \left.-\frac{1}{4}\left(1-\frac{1}{2(\log 2 Q+4 \log \alpha)}\right)^{4}\right]\left(1-\frac{1}{2(\log 2 Q+4 \log \alpha)}\right)^{-4}\left(1-\frac{9}{2(\log 2 Q+4 \log \alpha)}\right)^{-1} \\
& \left(1+\frac{1}{(\log 2 Q+4 \log \alpha)^{2}} \frac{1}{\left(1-\frac{1}{2(\log 2 Q+4 \log \alpha)}\right)}\right)^{2}
\end{aligned}
$$

For some typical value of the parameters $\mathrm{Q}=1$ and $\mathrm{E}=1$, and for large values of $\alpha$, it is obvious that $\rho_{\text {eff }}$ has a constant value. Therefore an observer on the brane will see an expanding inflating universe. It is interesting to see what happens for small values of $\alpha$. As $\alpha$ gets smaller, a term proportional to $\frac{1}{(\log \alpha)^{4}}$ starts to contribute to $\rho_{\text {eff }}$. Therefore the universe for small values of scale factor has a slow expanding inflationary phase which we call it "logarithmic inflationary" phase. For smaller value of $\alpha$ we cannot trust the solution which is reflected in the fact that $\rho_{\text {eff }}$ gets infinite. The behaviour of the effective energy density as a function of the scale factor is shown in Figure 1.

To have an idea how the slow inflationary phase proceeds, we can assume for the moment that the effective energy density scales as

$$
\frac{8 \pi}{3} \rho_{e f f}=\left(\frac{\dot{\alpha}}{\alpha}\right)^{2}=\frac{1}{(\log \alpha)^{p}}
$$

The solution of the above equation is

$$
\alpha=e^{t^{\left(\frac{2}{p+2}\right)}}
$$




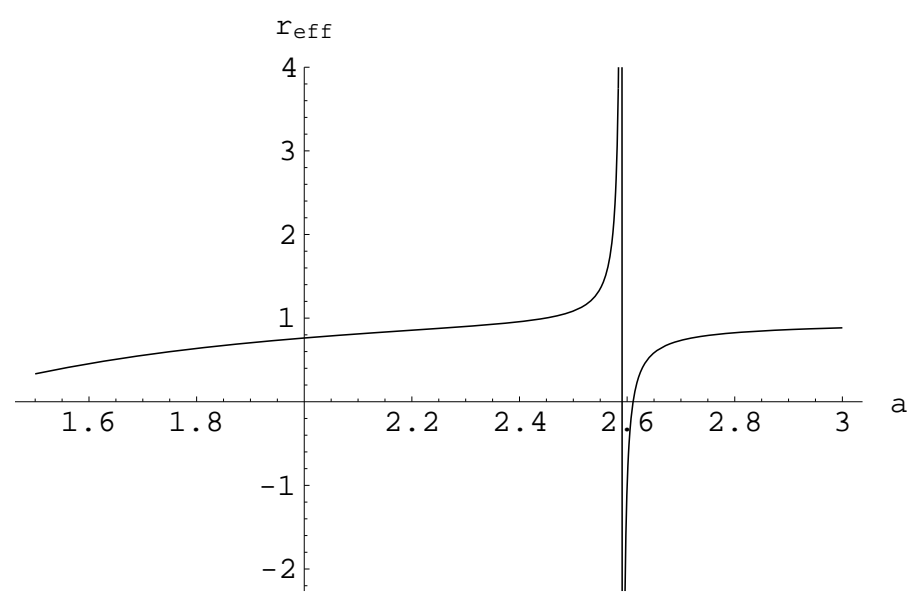

Figure 1: The induced energy density on the brane as a function of the brane scale factor.

Therefore we remain in an exponentially growing universe, but various values of $\mathrm{p}$ have the effect of making the universe to slow down its expansion. We note here that in order to estimate the behaviour and the duration of this "logarithmic inflationary" phase, we have to resolve the problem of the singularity.

Going now to IR using (3.27)-(3.30) we get for the metric components

$$
\begin{gathered}
g_{y y}=\frac{\sqrt{Q}}{16} 2^{-\frac{3}{4}}\left(1-\frac{1}{2 y}\right) \\
g=\frac{2^{-\frac{1}{4}}}{\sqrt{Q}} e^{-\frac{y}{2}}\left(1-\frac{9}{2 y}\right) \\
g_{s}=2^{-\frac{3}{4}} \sqrt{Q}\left(1+\frac{7}{2 y}\right)
\end{gathered}
$$

where now $y$ is defined by

$$
\rho=e^{y}
$$

Then the identification $g=\alpha^{2}$ using (4.14) gives again two solutions 


$$
\begin{gathered}
y_{1}=-\frac{9}{4 \log \alpha+\log \sqrt{2} Q} \\
y_{2}=-\log \sqrt{2} Q-4 \log \alpha+\frac{9}{\log \sqrt{2} Q+4 \log \alpha}
\end{gathered}
$$

For small $\alpha$ we keep from the solution $y_{2}$ of (4.18) the term $-\log \sqrt{2} Q-4 \log \alpha$. Using this solution we can calculate the $R R$ field

$$
C=-\frac{e^{-y}}{Q}-\frac{2}{Q} E i[-y]
$$

Then $\rho_{\text {eff }}$ becomes,

$$
\begin{aligned}
\frac{8 \pi}{3} \rho_{e f f}= & {\left[\left(-1-2 \frac{1}{\sqrt{2} Q \alpha^{4}} E i[\log \sqrt{2} Q+4 \log \alpha]+\frac{E}{\sqrt{2} \alpha^{4}}\right)^{2}-\right.} \\
& \left.\frac{1}{2}\left(1+\frac{9}{2(\log \sqrt{2} Q+4 \log \alpha)}\right)^{4}\right]\left(1+\frac{9}{2(\log \sqrt{2} Q+4 \log \alpha)}\right)^{-4} \\
& \left(1+\frac{1}{2(\log \sqrt{2} Q+4 \log \alpha)}\right)^{-1} \\
& \left(1-\frac{9}{2(4 \log \alpha+\log \sqrt{2} Q)^{2}} \frac{1}{\left(1-\frac{9}{2(\log \sqrt{2} Q+4 \log \alpha)}\right)}\right)^{2}
\end{aligned}
$$

As we can see, the above relation is the same as the energy density in the UV (relation (4.10)) up to some numerical factors, as expected. The difference is, that now it is valid for small $\alpha$. For small $\alpha$ first the term $\frac{1}{\alpha^{8}}$ dominates and then the term $\frac{1}{\alpha^{4}}$. As $\alpha$ increases the term $\frac{1}{(\log \alpha)^{4}}$ takes over and drives the universe to a slow inflationary expansion.

We will also discuss the cosmological behaviour of the IR solutions of [20] and [12]. Using (3.31)-(3.34) we have for the metric elements

$$
g_{\rho \rho}=e^{\left(\frac{1}{2}-\sqrt{5}\right) \rho} e^{-\frac{1}{4} e^{-2 \rho}+\frac{25}{2} e^{-\frac{4 \rho}{\sqrt{5}}}} \sqrt{Q}
$$




$$
\begin{gathered}
g=e^{-\frac{\rho}{2}} e^{\frac{1}{4} e^{-2 \rho}} \frac{1}{\sqrt{Q}} \\
g_{s}=e^{\left(\frac{1}{2}-\frac{1}{\sqrt{5}}\right) \rho} e^{-\frac{1}{4} e^{-2 \rho}+\frac{5}{2} e^{-\frac{4 \rho}{\sqrt{5}}}} \sqrt{Q}
\end{gathered}
$$

Then the equation $g=\alpha^{2}$ gives

$$
\rho=-4 \log \alpha-\log Q
$$

and the $R R$ field becomes

$$
C=\left(-2 e^{-2 \rho}-e^{-4 \rho}\right) \frac{1}{Q}
$$

Finally $\rho_{\text {eff }}$ becomes

$$
\begin{aligned}
\frac{8 \pi}{3} \rho_{e f f}= & \left(Q \alpha^{4}\right)^{-\sqrt{5}+\frac{1}{2}}\left[\left(-2 Q \alpha^{4}-Q^{3} \alpha^{12}+E \alpha^{-4}\right)^{2}-\left(1+Q^{2} \alpha^{8}\right)\right] \\
& \left(1-\frac{1}{4} Q^{2} \alpha^{8}+\frac{25}{2}\left(Q \alpha^{4}\right)^{\frac{4}{\sqrt{5}}}\right)^{-1}\left(1+Q^{2} \alpha^{8}\right)
\end{aligned}
$$

The above calculated effective energy density, in spite of its different form, has a similar

behaviour as (4.20). As $\alpha$ increases, various negative powers of $\alpha$ take over until the singularity is reached where positive powers of $\alpha$ dominate.

\section{Discussion}

We had followed a probe brane along a geodesic in the background of type 0 string. Assuming that the universe is described by a three-dimensional brane, we calculate the effective energy density which is induced on the brane because of this motion. We study this mirage matter as the brane-universe moves along the radial coordinate. 
In our previous work [10 we found, that the motion of the brane-universe in this particular background induces an inflationary phase on the brane. We made the analysis in the limited case where the dilaton and tachyon fields were constants. This assumption simplified the calculation because there is an exact solution of the equations of motion.

In this work we extend our study to a background where all the fields are functions of the radial coordinate. Then the problem becomes more complicated because there is no more an exact solution to the equations of motion. Nevertheless there are approximate solutions for large values of the radial coordinate, in the UV region and solutions for small values of the radial coordinate in the IR. In the UV the coupling of the theory is small, so we can trust the approximate solutions. In the IR, the coupling becomes strong but it was shown in the literature [20, 12], that all string corrections are small.

Using these solutions, we calculate the energy densities that are induced on the brane. What we find is that for large values of the scale factor as it is measured on the brane (large values of the radial coordinate) the universe enters a slow inflationary phase, in which the energy density is proportional to an inverse power of the logarithm of the scale factor. As the scale factor grows the induced energy density takes a constant value and the universe enters a normal exponential expansion. For small values of the scale factor the induced energy density scales as the inverse powers of the scale factor and then the logarithmic terms take over and the universe enters a slow exponential expansion.

The energy densities we calculated break down for some specific values of the scale factor. This is a reflection of the fact that the approximate solutions in the IR cannot be continued to the UV. To answer the question if there is a true phase of "logarithmic inflation" in which the universe inflates but with a slow rate, we must resolve the problem of singularities, where our theory breaks down. We are studying the problem numerically trying to solve the equations of motion numerically [12] and see if we can smoth out the singularities. Then we can apply our technics for calculating the effective energy density. 


\section{Acknowledgement}

We would like to thank A. Kehagias, E. Kiritsis and C. Bachas for valuable discussions. Work partially supported by the NTUA program Archimedes.

\section{Note Added}

While this work was written up to its final form, the reference [21] appeared where a similar problem was studied, and it was found that tachyonic background is less divergent than the one without tachyon.

\section{References}

[1] T.Regge and C.Teitelboim, Marcel Grossman Meeting on General Relativity,Trieste 1975, North Holland ;

V.A. Rubakov and M.E. Shaposhnikov, Do we live inside a domain wall? Phys. Lett.,B 125 (1983) 136.

[2] N.Arkani-Hamed, S.Dimopoulos and G.Dvali, The hierarchy problem and new dimensions at a millimeter, Phys. Lett. B 429(1998) 263 hep-ph/9803315; Phenomenology, astrophysics and cosmology of theories with submillimeter dimensions and TeV scale quantum gravity, Phys. Rev.D 59 (1999) 086004 [hep-ph/9807344;

I.Antoniadis, N.Arkani-Hamed, S.Dimopoulos and G.Dvali, New dimensions at a millimeter to a Fermi and superstrings at a TeV, Phys. Lett. B 436 (1998) 257 hepph/9804398 
[3] R.Sundrum, Effective field theory for a three-brane universe, Phys. Rev. D 59 (1999) 085009 hep-ph/9805471; Compactification for a three brane universe, Phys. Rev. D 59 (1999) 085010 hep-ph/9807348;

L.Randall and R.Sundrum, Out of this world supersymmetry breaking, Nucl. Phys. B.557 (1999) 79 [hep-th/9810155]; A large mass hierarchy from a small extra dimension, Phys. Rev. Lett. 83 (1999) 3370 hep-ph/9905221].

[4] J.Polchinski, Dirichlet branes and Ramond-Ramond charges, Phys. Rev. Lett. 75 (1995) 4724 hep-th/9510017

[5] P.Horava and E.Witten, Heterotic and type-I string dynamics from eleven dimensions, Nucl. Phys. B 460 (1996) 506 hep-th/9510209

[6] N.Kaloper and A.Linde, Inflation and large internal dimensions, Phys. Rev. D 59 (1999) 101303 hep-th/9811141;

N.Arkani-Hamed, S.Dimopoulos, N.Kaloper and J.March-Russell, Rapid asymmetric inflation and early cosmology in theories with submillimeter dimensions, hepph/9903224;

N.Arkani-Hamed, S.Dimopoulos, G.Dvali and N.Kaloper, Infinitely large new dimensions, hep-th/9907209;

N.Kaloper. Bent domain walls as brane-worlds, Phys. Rev. D60 (1999) 123506 [hepth/9905210.

P.Kanti, I.I.Kogan, K.A.Olive and M.Pospelov, Cosmological 3-Brane Solution, Phys. Lett. B468 (1999) 31 hep-ph/9909481

[7] P.Binetruy, C.Deffayet and D.Langlois, Nonconventional cosmology from a brane universe, [hep-th/9905012].

G.Dvali and S.H.Tye, Brane inflation, Phys. Lett. B450 (1999) 72 hep-ph/9812483; 
E.E.Flanagan, S.H.H.Tye and I.Wasserman, A cosmology of the brane world, hepph/9909373.

H.B.Kim and H.D.Kim, Inflation and Gauge Hierarchy in Randall-Sundrum Compactification, hep-th/9909053

C.Csaki, M.Graesser, C.Kolda and J.Terning, Cosmology of One Extra Dimension with Localized Gravity, Phys. Lett. B462 (1999) 34 [hep-ph/9906513

C.Csaki, M.Graesser, L.Randall and J.Terning, Cosmology of Brane Models with Radion Stabilization, hep-ph/9911406

[8] H.A.Chamblin and H.S.Reall, Dynamic dilatonic domain walls, hep-th/9903225; A.Chamblin, M.J/Perry and H.S.Reall, Non-BPS D8-branes and dynamic domain walls in massive IIA supergravities, J.High Energy Phys. 09 (1999) 014 hepth/9908047].

P.Kraus, Dynamics of Anti-de Sitter Domain Walls, JHEP 9912:011 (1999) [hepth/9910149]

[9] A.Kehagias and E.Kiritsis, Mirage cosmology, JHEP 9911:022 (1999) [hepth/9910174

[10] E.Papantonopoulos and I.Pappa, Type 0 Brane Inflation from Mirage Cosmology, hepth/0001183

[11] J.Y. Kim,Dilaton-driven brane inflation in type IIB string theory, hep-th/0004155

[12] R.Grena,S.Lelli,M.Maggiore,A.Rissone, Confinement, asymptotic freedom and renormalons in type 0 string duals, hep-th/0005213

[13] I.R.Klebanov and A.A.Tseytlin, D-Branes and Dual Gauge Theories in Type 0 Strings, Nucl.Phys. B 546(1999) 155 hep-th/9811035. 
[14] E. Papantonopoulos, Talk given at IX Marcel Grossman meeting, Rome July 2000, To appear in the proccedings

[15] I.R.Klebanov and A.Tseytlin, Asymptotic Freedom and Infrared Behavior in the type 0 String Approach to gauge theory, Nucl.Phys. B 547 (1999) 143 hep-th/9812089

[16] I.R.Klebanov Tachyon Stabilization in the AdS/CFT Correspondence [hepth/9906220]

[17] G.A.Diamandis, B.C.Georgalas and E.Papantonopoulos Tachyon Effects on the two-dimensional Black Hole Geometry Mod.Phys.Lett. A 10 (1995) 1277 hepth/9406028];

C.Chiou-Lahanas, G.A.Diamandis, B.C.Georgalas, X.N. Maintas E.Papantonopoulos ,Tachyon Field Quantization and Hawking Radiation Phys.Rev. D52 (1995) 5877 hep-th/9506059.

[18] G.A.Diamandis, B.C.Georgalas, N.E.Mavromatos and E.Papantonopoulos On 'Graceful Exit' from Inflationary Phase in two-dimensional Liouville String Cosmology Phys.Lett. B 461 (1999) 57 hep-th/9903045

[19] V.A. Kostelecky and M.J.Perry, Condensates and Singularities in String Theory, Nucl. Phys. B 414 (1994) 174 hep-th/9302120;

E.Raiten, Tachyon Condensates and String Theoretic Inflation, Nucl. Phys. B 416 (1994) 881 hep-th/9304048;

K. Behrndt, The Tachyon in a Linear Expanding Universe, Phys. Lett. B 301 (1993) 29 hep-th/9212099.

[20] J.A.Minahan, Asymptotic Freedom and Confinement from Type O String Theory, JHEP 9904:007 (1999) hep-th/9902074 
[21] Jin Young Kim, Brane inflation in tachyonic and non-tachyonic type OB string theories hep-th/0009175 